\title{
Valérie Cohen, Xavier Dunezat, Quand des chômeurs
} se mobilisent...

Rennes, Presses universitaires de Rennes, coll. « Res publica », 2018

\section{Charles Berthonneau}

\section{(2) OpenEdition}

\section{Journals}

\section{Édition électronique}

URL : http://journals.openedition.org/travailemploi/9687

DOI : $10.4000 /$ travailemploi.9687

ISSN : 1775-416X

\section{Éditeur}

DARES - Ministère du Travail

\section{Édition imprimée}

Date de publication : 2 décembre 2019

Pagination : 138-141

ISSN : 0224-4365

Référence électronique

Charles Berthonneau, "Valérie Cohen, Xavier Dunezat, Quand des chômeurs se mobilisent... », Travail et Emploi [En ligne], 160 | 2019, mis en ligne le 01 novembre 2020, consulté le 25 mars 2021. URL : http:// journals.openedition.org/travailemploi/9687 ; DOI : https://doi.org/10.4000/travailemploi.9687 


\section{Quand des chômeurs se mobilisent...}

\section{Valérie Cohen, Xavier Dunezat}

Rennes, Presses universitaires de Rennes, coll. « Res publica », 2018, 333 p.

Lu par Charles Berthonneau*

Le livre Quand des chômeurs se mobilisent... a pour premier mérite d'être le résultat d'une mise en commun originale de deux thèses menées séparément : l'une par Valérie Cohen sur l'organisation Agir contre le chômage (AC !), l'autre par Xavier Dunezat sur deux collectifs de chômeurs en Bretagne (Morlaix et Rennes). Ces deux sociologues ont réussi à traiter à partir d'un même cadre théorique les matériaux qu'ils ont récoltés lors de chacune de leur enquête ethnographique, évitant ainsi une simple juxtaposition de deux thèses différentes même si, bien évidemment, quand on entre plus précisément dans la démonstration, le découpage du plan reprend souvent une division selon leurs terrains respectifs. L'ouvrage est divisé en quatre parties : la première revient sur la manière dont se sont constituées historiquement les mobilisations de chômeurs, et le traitement dont elles ont fait l'objet dans les travaux de sociologie ; la deuxième explique les différentes trajectoires d'engagement selon les profils sociaux des militants qui composent les mouvements observés ; la troisième analyse finement l'organisation du travail militant et les rapports de domination qui en découlent ; enfin, la dernière partie propose des éclairages sur les différents effets de la participation aux mouvements sur les militants.

L'intérêt de l'ouvrage se situe à deux niveaux. D'une part, même si la littérature sur le sujet - que les auteurs passent largement en revue - est abondante, il apporte beaucoup à la compréhension des dynamiques des mobilisations de chômeurs qui, bien qu'elles soient moins visibles que dans les années 1990 (le mouvement de l'hiver 1997-1998 a, par exemple, été particulièrement marquant), n'en demeurent pas moins actives, notamment au niveau local et pour défendre au quotidien les chômeurs face aux autorités administratives. D'autre part, il dépasse largement le seul sujet des mobilisations de chômeurs pour offrir une boîte à outils conceptuels et une méthode d'enquête utiles pour l'étude de n'importe quel mouvement social. C'est bien ce deuxième niveau qui constitue la principale force de l'ouvrage. Ainsi, alors que certains regrettent parfois que l'ethnographie livre des analyses trop microsociologiques, valables uniquement pour un cas d'étude précis, V. Cohen et X. Dunezat proposent une réelle montée

\footnotetext{
* Laboratoire d'économie et de sociologie du travail (LEST - UMR 7317), Aix-Marseille Université.
} 
en généralité qui permet de tirer des enseignements à l'échelle de l'ensemble des mouvements sociaux.

Considérant que l'angle de l' « improbabilité », généralement utilisé pour étudier les mobilisations de groupes dits à «faibles ressources », enferme ces derniers dans une spécificité qu'ils estiment injustifiée, et plutôt que de se contenter d'étudier les conditions de possibilité de ces luttes, les auteurs préfèrent s'intéresser à la manière dont elles sont structurées dans leur dimension routinière, c'est-à-dire comment est organisée concrètement la « division du travail militant». En somme, il s'agit de reprendre des questions classiques de la sociologie des mouvements sociaux, tout en tâchant d'expliquer «en quoi la condition de chômeur [colore] l'action collective » (p. 17). De leur démarche résultent de nombreux apports, que nous résumerons en trois points.

Premièrement, leur approche évite de réifier les groupes mobilisés derrière une catégorie faussement homogène, comme celle de « chômeurs », pour rendre compte de la diversité des sous-groupes qui constituent ces mobilisations. Leur catégorisation dépasse le clivage trop réducteur entre « militants par conscience » et «bénéficiaires » (clivage propre à la sociologie des mobilisations dites « improbables ») pour réussir à dégager les différents facteurs qui, au sein de la mobilisation, s'avèrent clivants : les rapports sociaux (de sexe, de classe, et d'âge principalement) comme l'expérience - ou le manque d'expérience - de militantisme sont analysés. Ils proposent ainsi une « cartographie des pôles » (p. 107) - « syndicaliste », « anarchiste », « chômeur » dont la cohérence repose autant sur des caractéristiques sociales que sur des modes différents d'entrée dans l'engagement et de participation, ou encore sur des rapports différenciés au travail et au chômage. Ils ont notamment le mérite de ne pas passer à côté de « figures intermédiaires » moins visibles, à savoir principalement des femmes qui prennent en charge les tâches administratives et logistiques, et assurent le lien entre ces différents pôles.

Cependant, alors même qu'ils ont pour objectif de mettre en lumière celles et ceux qui, dans la sociologie des mobilisations, sont les plus invisibilisées, c'est-à-dire celles et ceux qui disposent du moins de capital militant, on reste justement sur notre faim quant à l'explication des conditions d'engagement des plus précaires dans l'action collective. Si on comprend effectivement que ces chômeurs sont en quelque sorte déjà «mobilisés »-pour reprendre le terme que les auteurs utilisent - avant d'entrer dans le mouvement à travers leurs différentes « pratiques de subsistances » (p. 135) auprès des multiples guichets qui permettent de rendre leur situation plus supportable, les conditions concrètes du passage à l'acte de mobilisation sont peu explicitées. Tout au plus est souligné le rôle du « travail relationnel » (p. 145) auquel se livrent des militants plus aguerris pour que les plus précaires restent mobilisés. On souhaiterait en savoir davantage sur le travail de légitimation de l'action collective sans lequel l'engagement de ces chômeurs est impossible étant donné qu'ils n'ont jamais participé à des mobilisations ; en particulier, comment les militants leur transmettent-ils dans la durée des grilles de lecture «politisées » de la réalité ? 
Deuxièmement, si ce ne sont pas les seuls ni les premiers à utiliser le concept de travail militant, $\mathrm{V}$. Cohen et $\mathrm{X}$. Dunezat en proposent ici un véritable approfondissement et en montrent toute la portée heuristique. Comparant le militantisme à n'importe quel travail, ils s'inspirent des analyses généralement utilisées pour étudier des organisations capitalistes du travail. Ils opposent ainsi la notion de «travail prescrit » (qui recouvre les activités légitimes, c'est-à-dire celles qui font l'objet d'une formalisation, d'une ritualisation, par exemple l'organisation d'une assemblée générale) à celle de « travail réel » (autrement dit l'ensemble des tâches qui contribuent à faire tenir les mobilisations ; elles englobent autant le travail prescrit que des activités informelles, moins visibles et légitimes, plus souvent effectuées par des femmes, comme la gestion des repas), et la notion de « travail séparé » (division du travail militant selon laquelle chaque «pôle » se spécialise sur une tâche) à celle de «travail collectif » (division du travail militant reposant sur une appropriation collective des différentes tâches). Une telle démarche permet de comprendre concrètement et finement les ressorts des rapports de domination internes aux mouvements, au sein desquels la prise en charge des différentes tâches nécessaires pour faire tenir la mobilisation obéit à une logique de hiérarchisation.

De plus, le fait même de s'intéresser à l'organisation du travail militant éclaire sous un jour nouveau les raisons de l'engagement. D'après les auteurs, les chômeurs s'engagent s'ils pensent trouver du travail militant disponible nécessitant les mêmes savoir-faire et dispositions que ceux qu'ils ont déjà. Alors que les travaux de recherche étudient plutôt d'ordinaire comment des dispositions sont incorporées au cours d'une socialisation donnée, V. Cohen et X. Dunezat analysent la manière dont un fonctionnement organisationnel permet ou non l'actualisation de dispositions mobilisées précédemment. C'est ainsi que des chômeurs pourtant sans aucune expérience militante antérieure peuvent trouver leur place dans la mobilisation en participant aux occupations de nuit, parce qu'ils ont déjà été habitués à tenir ces rythmes horaires et ces exigences physiques au cours de leurs anciens emplois.

Selon V. Cohen et X. Dunezat, les raisons de l'engagement, du défaut d'engagement ou du désengagement, sont donc moins à chercher du côté des dispositions des chômeurs et des chômeuses que du côté du fonctionnement des organisations, ce qui reste encore trop peu étudié dans la littérature existante ; s'y intéresser suppose de prêter plus d'attention aux observations, alors que bon nombre d'enquêtes s'appuient essentiellement sur des entretiens. Leur travail montre très bien que si les classes populaires restent en dehors (ou en retrait) de l'action militante, ce n'est pas tant parce qu'elles manqueraient intrinsèquement des ressources nécessaires, mais plutôt parce que le fonctionnement des organisations privilégie surtout les ressources des classes les plus diplômées.

Troisièmement, leur travail s'inscrit dans les repositionnements de la sociologie des mouvements sociaux : alors que celle-ci s'est longtemps concentrée sur la seule étude des conditions d'entrée dans l'engagement, elle cherche désormais à comprendre les effets de l'action collective. Les auteurs soulignent ainsi que, pour la population 
qu'ils étudient, ordinairement exposée à une vulnérabilité sociale et à des formes de désaffiliation, participer à une action militante a des effets en termes d'émancipation individuelle et d'intégration sociale. Mais l'originalité de leur travail repose sur la mise en lumière des processus de disqualification subis par les militants les plus précaires (les «SDF », p. 264), qui, aux yeux de ceux disposant d'une condition sociale plus stabilisée, ne correspondent pas à des figures légitimes de militants. Les mobilisations ont donc aussi pour conséquence de produire et de renforcer une stigmatisation des plus éloignés des normes sociales dominantes. Contre l'idée reçue selon laquelle l'action collective engendre nécessairement des effets positifs, V. Cohen et X. Dunezat questionnent véritablement le fonctionnement des organisations militantes en mettant au jour les tensions qui les traversent. 